ISSN 1112-9867

http://www.jfas.info

\title{
A GREEN AND EFFICIENT METHOD FOR THE SYNTHESIS OF HOMODIMERIC (B-DICARBONYL) ARYLMETHANES AND DIHYDROPYRIDINE FROM DIMEDONE IN WATER
}

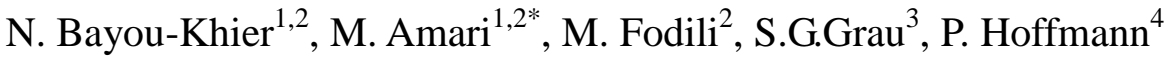 \\ ${ }^{1}$ Laboratoire de Chimie Organique et des Substances Naturelles, Université Ziane Achour, \\ Djelfa, Algerie. \\ ${ }^{2}$ Faculté de Chimie - USTHB - BP32, El-Alia, 16111 Bab Ezzouar, Alger, Algerie \\ ${ }^{3}$ C.M.R.M.D.T. (idm), Université de Valence, Burjassot, Valence, Espagne. \\ ${ }^{4}$ Laboratoire de Synthèse et Physico-Chimie de Molécules d'Intérêt Biologique, UMR 5068, \\ Université Paul Sabatier, 118 route de Narbonne, 31062 Toulouse, Cedex 4, France
}

Received: 30 May 2016 / Accepted: 19 August 2016 / Published online: 01 September 2016

\begin{abstract}
A direct method has been developed for the synthesis of the dihydropyridine ring system by means of Michael reaction. The reaction of dimedone with 1.0 equiv. of amines in water provides intermediate product, which allowed dihydropyridine derivatives by intramolecular cyclization in various yields. Of particular interest is the use of the water as solvent of reaction and in absence of catalyst. Also these operating conditions protect the environment and economic points of view.
\end{abstract}

Keywords aqueous synthesis; bioactivity; dihydropyridine; dimedone; green method; selective conditions.

Author Correspondence, e-mail: amarimod@ hotmail.fr

doi: http://dx.doi.org/10.4314/jfas.v8i3.16 


\section{INTRODUCTION}

Homodimeric ( $\beta$-dicarbonyl) arylmethane derivatives have attracted considerable attention since they exhibit potent bioactivity, like anti-inflammatory, antibiotic and anti-oxydant principle [1-3]. Several syntheses have been developed for the preparation of these homodimeric compounds. These routes usually involved condensation of aldehydes with electron-rich carbon nucleophiles (enols, etheroaromatics) [4-6].

The molecule dimedone $\underline{\mathbf{1}}$, known for a long time, has been scarcely used in the synthesis of heterocyclic structures [7-13]. According to the literature, the homodimer $\underline{\mathbf{3}}$ of dimedone has been obtained in various organic solvents like acetic acid and in the presence of toxic and not toxic catalysts such as cyanuric chloride and triethylamine.

In this work, we have obtained selectively, the homodimer $\underline{\mathbf{3}}$ of dimedone along with the xanthene structure $\underline{4}$ by a green method as we have discover that in aqueous solution, no catalysis is required and products are isolated by filtration avoiding the use of any organic solvent (Scheme 1). Under these clean operating conditions, we have improved yields and master their reaction times compared to those cited in the literature [14]. Our interest in this type of structure is mainly based on its functional groups likely to give new heterocyclic structures. In particular, the action of primary amines on $\underline{\mathbf{3}}$ forms a structure dihydropyridine (DHP) which exhibit an interesting functionalization.

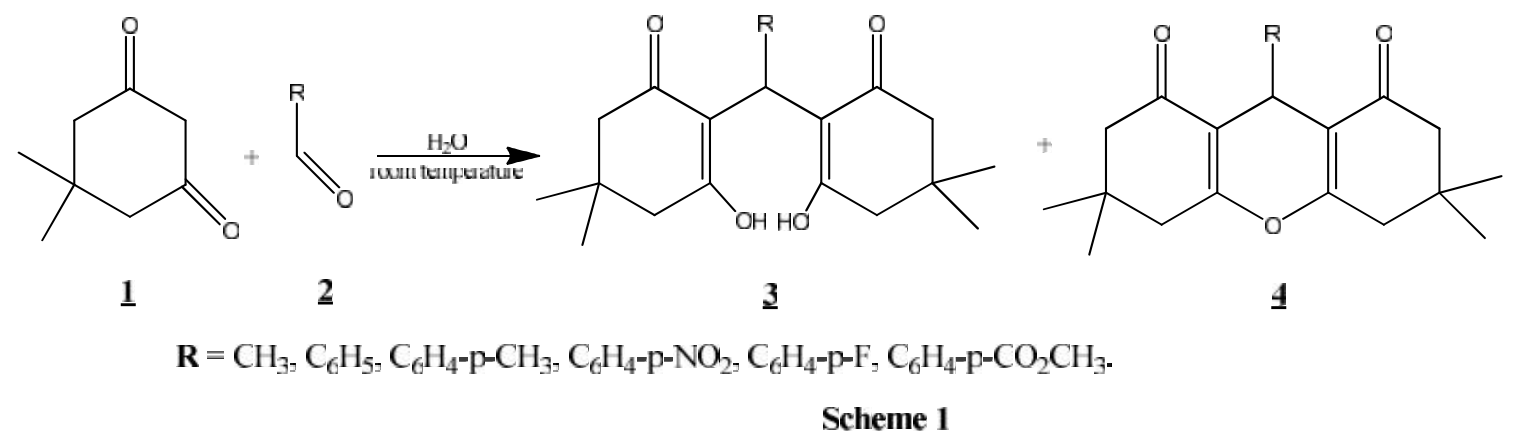

\section{RESULTS AND DISCUSSION}

When two equivalents of dimedone $\underline{\mathbf{1}}$ (5,5-Dimethyl-1,3-cyclohexanedione) and one equivalent of aldehyde $\underline{\mathbf{2}}$ are dissolved in dichloromethane under magnetic stirring, at room temperature or heated under reflux, only the structure $\underline{4}$ was obtained. The same result is obtained in a 50:50 mixture of $\mathrm{CH}_{2} \mathrm{Cl}_{2} / \mathrm{H}_{2} \mathrm{O}$. On the other hand, the use of $\mathrm{H}_{2} \mathrm{O}$ as a solvent drives the reaction selectively to $\underline{\mathbf{3}}$ or $\underline{\mathbf{4}}$ depending on the temperature and time. Thus, heating 
at $100^{\circ} \mathrm{C}$, promotes the formation of the structure $\underline{4}$. At room temperature, we obtained selectively $\underline{\mathbf{3}}$ and $\underline{\mathbf{4}}$, depending on the reaction time.

Table 1 : Conditions of formation and physical data of $\underline{\mathbf{3}}$ and $\underline{\mathbf{4}}$ in water at $25^{\circ} \mathrm{C}$.

\begin{tabular}{|c|c|c|c|c|}
\hline Compounds & $\mathrm{R}$ & Time(h) & Yield (\%) & $\mathrm{mp}\left({ }^{\circ} \mathrm{C}\right)$ \\
\hline$\underline{\mathbf{3 a}}$ & $\mathrm{CH}_{3}$ & 3 & 55 & $129-131$ \\
\hline$\underline{3 \mathbf{b}}$ & $\mathrm{C}_{6} \mathrm{H}_{5}$ & 2.30 & 83 & $188-190$ \\
\hline$\underline{3 c}$ & $\mathrm{C}_{6} \mathrm{H}_{4} \mathrm{CH}_{3}$ & // & 61 & $131-133$ \\
\hline$\underline{\mathbf{3 d}}$ & $\mathrm{C}_{6} \mathrm{H}_{4} \mathrm{NO}_{2}$ & // & 78 & 192-194 \\
\hline$\underline{3 e}$ & $\mathrm{C}_{6} \mathrm{H}_{4} \mathrm{~F}$ & // & 86 & $165-167$ \\
\hline$\underline{\mathbf{3 f}}$ & $\mathrm{C}_{6} \mathrm{H}_{4} \mathrm{CO}_{2} \mathrm{CH}_{3}$ & // & 89 & 197-199 \\
\hline$\underline{3 g}$ & $\mathrm{C}_{6} \mathrm{H}_{4} \mathrm{OH}$ & // & 75 & 209-211 \\
\hline$\underline{4 \mathbf{a}}$ & $\mathrm{CH}_{3}$ & 5 & 67 & $184-186$ \\
\hline$\underline{4 b}$ & $\mathrm{C}_{6} \mathrm{H}_{5}$ & 4.30 & 88 & 196-198 \\
\hline$\underline{4 c}$ & $\mathrm{C}_{6} \mathrm{H}_{4} \mathrm{CH}_{3}$ & $/ /$ & 74 & $219-221$ \\
\hline$\underline{\mathbf{4 d}}$ & $\mathrm{C}_{6} \mathrm{H}_{4} \mathrm{NO}_{2}$ & // & 96 & $231-233$ \\
\hline$\underline{4 e}$ & $\mathrm{C}_{6} \mathrm{H}_{4} \mathrm{~F}$ & // & 93 & $213-215$ \\
\hline$\underline{4 f}$ & $\mathrm{C}_{6} \mathrm{H}_{4} \mathrm{CO}_{2} \mathrm{CH}_{3}$ & // & 97 & $229-231$ \\
\hline $4 \mathrm{~g}$ & $\mathrm{C}_{6} \mathrm{H}_{4} \mathrm{OH}$ & // & 81 & $241-243$ \\
\hline
\end{tabular}

The formation of $\underline{\mathbf{3}}$ and $\underline{\mathbf{4}}$, can be explained by the condensation of the enolic form of the dimedone on aldehyde, according to the reaction of Knoevenagel, followed by an addition on a second molecule of dimedone, according to the reaction of Michael (Scheme 1).

In $\mathrm{CH}_{2} \mathrm{Cl}_{2}$ or $\mathrm{CH}_{2} \mathrm{Cl}_{2} / \mathrm{H}_{2} \mathrm{O}$ at $50 \%$, the interactions $\mathrm{OH}---\mathrm{O}=\mathrm{C}$, present in $\underline{\mathbf{3}}$, are weak and lead in each case to structure $\underline{4}$. On the other hand in $\mathrm{H}_{2} \mathrm{O}$, the hydrogen bonds $\mathrm{OH}---\mathrm{O}=\mathrm{C}$ are accentuated and consolidated by the polarity of $\mathrm{H}_{2} \mathrm{O}$. These interactions support the formation of $\underline{\mathbf{3}}$. But energy brought, in the course of the reaction time under the effect of agitation, is favorable to the rupture of the hydrogen bonds, to transform $\underline{\mathbf{3}}$ into $\underline{\mathbf{4}}$, by an intramolecular ceto-enolic condensation.

Since our objective is to obtain new heterocyclic molecules, as the dihydropyridine $\underline{\mathbf{5}}$, potentially active in various fields of applications [15-17]. As a first step, we valued structure 
$\underline{\mathbf{3}}$ in the synthesis of new heterocyclic molecules, standard dihydropyridine, by subjecting it to the action of two monoamines (Scheme 2).

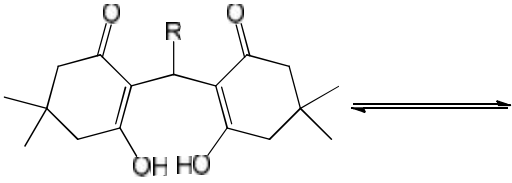

3e, $\mathrm{R}=\mathrm{C}_{6} \mathrm{H}_{5}-\mathrm{p}-\mathrm{F}$

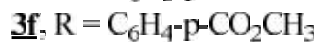
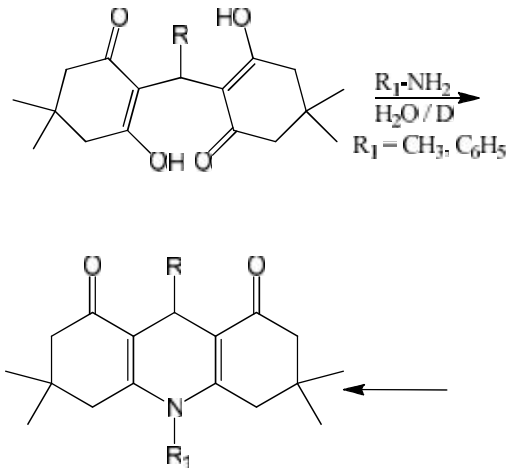

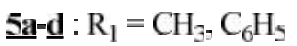

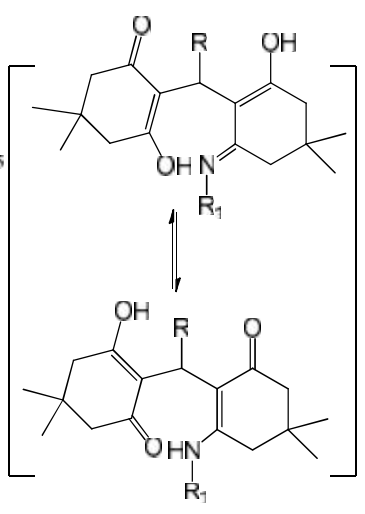

Scheme 2

Under clean operating conditions i.e. $\mathrm{H}_{2} \mathrm{O}$ at $100{ }^{\circ} \mathrm{C}$, we easily isolated the structure dihydropyridine $\underline{\mathbf{5}}$, according to the reaction of Hantzsch [18].

All compounds $\underline{\mathbf{5}}$ were characterized by the various spectroscopic methods. Their physical properties are summarized in table 2.

Table 2 : Conditions of formation and physical data of $\underline{\mathbf{5}}$ at $100{ }^{\circ} \mathrm{C}$.

\begin{tabular}{|c|c|c|c|c|c|}
\hline Compounds & $\mathrm{R}$ & $\mathrm{R}_{1}$ & Time (h) & Yield $(\%)$ & $\mathrm{mp}\left({ }^{\circ} \mathrm{C}\right)$ \\
\hline$\underline{\mathbf{5}}$ & $\mathrm{C}_{6} \mathrm{H}_{4}-\mathrm{F}$ & $\mathrm{CH}_{3}$ & 5 & 45 & $215-217$ \\
\hline$\underline{\mathbf{5 b}}$ & $/ /$ & $\mathrm{C}_{6} \mathrm{H}_{5}$ & 4 & 63 & $231-233$ \\
\hline$\underline{\mathbf{5}}$ & $\mathrm{C}_{6} \mathrm{H}_{4}-\mathrm{CO}_{2} \mathrm{CH}_{3}$ & $\mathrm{CH}_{3}$ & 6 & 51 & $223-225$ \\
\hline$\underline{\mathbf{5 d}}$ & $/ /$ & $\mathrm{C}_{6} \mathrm{H}_{5}$ & 3 & 67 & $239-241$ \\
\hline
\end{tabular}

The amine's basicity allows, in a first step, the breaking of the hydrogen bond $\mathrm{OH}---\mathrm{O}=\mathrm{C}$ by electronic interaction $\mathrm{N}---\mathrm{H}$, according to the acid-base properties of Lewis. In a second step, the primary amine reacts with the carbonyl present in $\underline{\mathbf{3}}$ to form the corresponding imine. Finally, the equilibrium enol-ketone and imine-enamine present in the reactional intermediary, leads, by interaction ketone-enamine to a heterocyclization of the structure dihydropyridine $\underline{\mathbf{5}}$.

\section{EXPERIMENTAL}

All chemicals were obtained from Aldrich. Melting points were taken on a Thomas Hoover apparatus and are uncorrected. Nuclear magnetic resonance spectra were obtained with a Bruker AC 300 at $300 \mathrm{MHz}\left({ }^{1} \mathrm{H}\right)$ or $75 \mathrm{MHz}\left({ }^{13} \mathrm{C}\right)$. The chemical shifts are reported in ppm 
( $\delta$-scale) relative to internal TMS and coupling constants are reported in Hertz $(\mathrm{Hz})$. The impact ionization mass spectra were recorded on a Nermag R10-10C at $70 \mathrm{eV}$.

\section{General procedure for synthesis of $\underline{3}$ and $\underline{4}$}

In $20 \mathrm{ml}$ water, a dimedone $\underline{\mathbf{1}}\left(2.10^{-2} \mathrm{~mol}\right)$ was reacted with aldehydes $\underline{\mathbf{2}}$ (a to $\left.\mathbf{g}\right)\left(10^{-2} \mathrm{~mol}\right)$. The mixture was stirred at room temperature 2 hours or refluxed for $4 \mathrm{~h}$ under magnetic stirring. We obtained selectively, after filtration and dry vacuum the corresponding compound $\underline{\mathbf{3}}$ (a to $\mathbf{g})$ or $\underline{\mathbf{4}}$ (a to $\mathbf{g})$.

\section{General procedure for synthesis of $\underline{\mathbf{5}}$}

Homodimer compound $\underline{\mathbf{3}}\left(10^{-2} \mathrm{~mol}\right)$ in $20 \mathrm{ml}$ of water, reacted with monoamines $\left(10^{-2} \mathrm{~mol}\right)$. The mixture was refluxed for $4 \mathrm{~h}$ at $100{ }^{\circ} \mathrm{C}$, under magnetic stirring. After filtration and dry vacuum, we obtained the corresponding compounds $\underline{\mathbf{5}}$ ( $\underline{\mathbf{a}}$ to $\underline{\mathbf{d}})$.

\section{2,2'-(ethane-1,1-diyl)bis(3-hydroxy-5,5-dimethylcyclohex-2-enone) $\underline{3 a}$}

Yield: 55\%; ${ }^{1} \mathrm{H}$ NMR $\left(300 \mathrm{MHz}, \mathrm{CDCl}_{3}\right): \delta(\mathrm{ppm}) 1.07\left(\mathrm{~s}, 6 \mathrm{H}, 2 \mathrm{CH}_{3}\right), 1.12\left(\mathrm{~s}, 6 \mathrm{H}, 2 \mathrm{CH}_{3}\right)$, $1.29\left(\mathrm{~d}, \mathrm{~J}=6.1 \mathrm{~Hz}, 3 \mathrm{H}, \mathrm{CH}_{3}\right), 2.13-2.18\left(\mathrm{~m}, 8 \mathrm{H}, 4 \mathrm{CH}_{2}\right), 4.89$ (d, J=6.1Hz, 1H, CH), 10.98 (s, $1 \mathrm{H}, \mathrm{OH}) ;{ }^{13} \mathrm{C} \mathrm{NMR}\left(75 \mathrm{MHz}, \mathrm{CDCl}_{3}\right): \delta(\mathrm{ppm}) 21\left(\mathrm{CH}_{3}\right), 25\left(2 \mathrm{CH}_{3}\right), 28\left(2 \mathrm{CH}_{3}\right), 30(2 \mathrm{Cq})$, $31(\mathrm{CH}), 43\left(2 \mathrm{CH}_{2}\right), 45\left(2 \mathrm{CH}_{2}\right), 113(2 \mathrm{Cq}), 161(2=\mathrm{C}-\mathrm{OH}), 187(2 \mathrm{C}=\mathrm{O})$. E.I. $(70 \mathrm{eV}), \mathrm{m} / \mathrm{z}(\%)$ : $M\urcorner^{+.}=306(14)$

\section{2,2'-(phenylmethylene)bis(3-hydroxy-5,5-dimethylcyclohex-2-enone) $\underline{3 b}$}

Yield: 83\%; ${ }^{1} \mathrm{H}$ NMR $\left(300 \mathrm{MHz}, \mathrm{CDCl}_{3}\right): \delta(\mathrm{ppm}): 1.04\left(\mathrm{~s}, 6 \mathrm{H}, 2 \mathrm{CH}_{3}\right), 1.11\left(\mathrm{~s}, 6 \mathrm{H}, 2 \mathrm{CH}_{3}\right)$, 2.31-2.39 (m, 8H, 4CH $), 5.39(\mathrm{~s}, 1 \mathrm{H}, \mathrm{CH}), 7.03-7.15\left(\mathrm{~m}, 5 \mathrm{H}, \mathrm{CH}_{\text {arom }}\right), 11.81(\mathrm{~s}, 1 \mathrm{H}, \mathrm{OH})$; ${ }^{13} \mathrm{C} \mathrm{NMR}\left(75 \mathrm{MHz}, \mathrm{CDCl}_{3}\right): \delta(\mathrm{ppm}) 28\left(2 \mathrm{CH}_{3}\right), 32\left(2 \mathrm{CH}_{3}\right), 33(2 \mathrm{Cq}), 34(\mathrm{CH}), 48\left(2 \mathrm{CH}_{2}\right)$, 51 $\left(2 \mathrm{CH}_{2}\right), 126-127-129-158\left(6 \mathrm{C}_{\text {arom }}\right), 116(2 \mathrm{Cq}), 163(2=\mathrm{C}-\mathrm{OH}), 188(2 \mathrm{C}=\mathrm{O})$. E.I. $(70 \mathrm{eV}), \mathrm{m} / \mathrm{z}$ $(\%): M\urcorner^{+.}=368(40), 229(100)$

\section{2,2'-(p-tolylmethylene)bis(3-hydroxy-5,5-dimethylcyclohex-2-enone) $\underline{3 c}$}

Yield: 61\%; ${ }^{1} \mathrm{H}$ NMR $\left(300 \mathrm{MHz}, \mathrm{CDCl}_{3}\right): \delta(\mathrm{ppm}): 1.07\left(\mathrm{~s}, 6 \mathrm{H}, 2 \mathrm{CH}_{3}\right), 1.20\left(\mathrm{~s}, 6 \mathrm{H}, 2 \mathrm{CH}_{3}\right)$, $2.27\left(\mathrm{~s}, 3 \mathrm{H}, \mathrm{CH}_{3}\right), 2.37-2.41\left(\mathrm{~m}, 8 \mathrm{H}, 4 \mathrm{CH}_{2}\right), 5.48(\mathrm{~s}, 1 \mathrm{H}, \mathrm{CH}), 6.97-7.04\left(\mathrm{~m}, 4 \mathrm{H}, \mathrm{CH}_{\text {arom }}\right)$, $11.87(\mathrm{~s}, 1 \mathrm{H}, \mathrm{OH}) ;{ }^{13} \mathrm{C} \mathrm{NMR}\left(75 \mathrm{MHz}, \mathrm{CDCl}_{3}\right): \delta(\mathrm{ppm}) 26\left(\mathrm{CH}_{3 \text { arom }}\right), 27\left(2 \mathrm{CH}_{3}\right), 29\left(2 \mathrm{CH}_{3}\right)$, $30(2 \mathrm{Cq}), 31(\mathrm{CH}), 44\left(2 \mathrm{CH}_{2}\right), 45\left(2 \mathrm{CH}_{2}\right), 112(2 \mathrm{Cq}), 127-128-134-156\left(6 \mathrm{C}_{\mathrm{arom}}\right), 161(2=\mathrm{C}-\mathrm{OH})$, 191(2C=O). E.I. $(70 \mathrm{eV}), \mathrm{m} / \mathrm{z}(\%): \mathrm{M}^{+\cdot}=382(33), 243$ (59). 


\section{2,2'-((4-nitrophenyl)methylene)bis(3-hydroxy-5,5-dimethylcyclohex-2-enone) $\underline{\mathbf{3 d}}$}

Yield: $78 \%$; ${ }^{1} \mathrm{H}$ NMR $\left(300 \mathrm{MHz}, \mathrm{CDCl}_{3}\right): \delta(\mathrm{ppm}): 1.12\left(\mathrm{~s}, 6 \mathrm{H}, 2 \mathrm{CH}_{3}\right), 1.25\left(\mathrm{~s}, 6 \mathrm{H}, 2 \mathrm{CH}_{3}\right)$, 2.35-2.49 (m, 8H, 4CH $), 5.54(\mathrm{~s}, 1 \mathrm{H}, \mathrm{CH}), 7.19-8.01\left(\mathrm{~m}, 4 \mathrm{H}, \mathrm{CH}_{\text {arom }}\right), 11.87$ (s, $\left.1 \mathrm{H}, \mathrm{OH}\right)$; ${ }^{13} \mathrm{C} \mathrm{NMR}\left(75 \mathrm{MHz}, \mathrm{CDCl}_{3}\right): \delta(\mathrm{ppm}) 28\left(2 \mathrm{CH}_{3}\right), 32\left(2 \mathrm{CH}_{3}\right), 34(2 \mathrm{Cq}), 35(\mathrm{CH}), 49\left(2 \mathrm{CH}_{2}\right)$, 52(2 $\left.\mathrm{CH}_{2}\right), 116(2 \mathrm{Cq}), 123-129-141-157\left(6 \mathrm{C}_{\text {arom }}\right), 164(2=\mathrm{C}-\mathrm{OH}), 189(2 \mathrm{C}=\mathrm{O})$. E.I. $(70 \mathrm{eV}), \mathrm{m} / \mathrm{z}$ $(\%): M\urcorner^{+.}=413(27), 275(65)$.

\section{2,2'-((4-fluorophenyl)methylene)bis(3-hydroxy-5,5-dimethylcyclohex-2-enone) $\underline{\mathbf{3 e}}$}

Yield: $86 \%$; ${ }^{1} \mathrm{H}$ NMR $\left(300 \mathrm{MHz}, \mathrm{CDCl}_{3}\right): \delta(\mathrm{ppm}): 1.01\left(\mathrm{~s}, 6 \mathrm{H}, 2 \mathrm{CH}_{3}\right), 1.22\left(\mathrm{~s}, 6 \mathrm{H}, 2 \mathrm{CH}_{3}\right)$, 2.40-2.43 (m, 8H, 4CH $), 5.48(\mathrm{~s}, 1 \mathrm{H}, \mathrm{CH}), 6.92-7.03\left(\mathrm{~m}, 4 \mathrm{H}, \mathrm{CH}_{\text {arom }}\right), 11.88(\mathrm{~s}, 1 \mathrm{H}, \mathrm{OH})$; ${ }^{13} \mathrm{C} \mathrm{NMR}\left(75 \mathrm{MHz}, \mathrm{CDCl}_{3}\right): \delta(\mathrm{ppm}) 27\left(2 \mathrm{CH}_{3}\right), 30\left(2 \mathrm{CH}_{3}\right), 31(2 \mathrm{Cq}), 32(\mathrm{CH}), 46\left(2 \mathrm{CH}_{2}\right)$, 47(2 $\left.\mathrm{CH}_{2}\right), 115(2 \mathrm{Cq}), 117-128-133-159\left(6 \mathrm{C}_{\text {arom }}\right), 162(2=\mathrm{C}-\mathrm{OH}), 189(2 \mathrm{C}=\mathrm{O})$. C.I. $\left(\mathrm{NH}_{3}\right)$ : $\mathrm{MH}^{7^{+}}=387(23 \%), 244(5 \%)$.

\section{Methyl- 4-(bis(2-hydroxy-4,4-dimethyl-6-oxocyclohex-1-en-1-yl)methyl)benzoate $\underline{3 f}$}

Yield: $89 \%$; ${ }^{1} \mathrm{H}$ NMR $\left(300 \mathrm{MHz}, \mathrm{CDCl}_{3}\right): \delta(\mathrm{ppm}): 1.10\left(\mathrm{~s}, 6 \mathrm{H}, 2 \mathrm{CH}_{3}\right), 1.23\left(\mathrm{~s}, 6 \mathrm{H}, 2 \mathrm{CH}_{3}\right)$, 2.34-2.45 (m, 8H, 4CH 2$), 3.88\left(\mathrm{~s}, 3 \mathrm{H}, \mathrm{O}-\mathrm{CH}_{3}\right), 5.53(\mathrm{~s}, 1 \mathrm{H}, \mathrm{CH}), 7.16-7.94\left(\mathrm{~m}, 4 \mathrm{H}, \mathrm{CH}_{\text {arom }}\right)$, $11.84(\mathrm{~s}, 1 \mathrm{H}, \mathrm{OH}) ;{ }^{13} \mathrm{C} \mathrm{NMR}\left(75 \mathrm{MHz}, \mathrm{CDCl}_{3}\right): \delta(\mathrm{ppm}) 27\left(2 \mathrm{CH}_{3}\right), 32\left(2 \mathrm{CH}_{3}\right), 34(2 \mathrm{Cq}), 35$ $(\mathrm{CH}), 48\left(2 \mathrm{CH}_{2}\right), 49\left(\mathrm{O}-\mathrm{CH}_{3}\right), 53\left(2 \mathrm{CH}_{2}\right), 118(2 \mathrm{Cq}), 129-130-131-159\left(6 \mathrm{C}_{\text {arom }}\right), 165(2=\mathrm{C}-\mathrm{OH})$, 167(Cq), 192(2C=O). E.I. $(70 \mathrm{eV}), \mathrm{m} / \mathrm{z}(\%): \mathrm{M}\urcorner^{+.}=436(15), 298(64)$.

\section{2,2'-((4-hydroxyphenyl)methylene)bis(3-hydroxy-5,5-dimethylcyclohex-2-enone) $\underline{\mathbf{3 g}}$}

Yield: $75 \%$; ${ }^{1} \mathrm{H}$ NMR $\left(300 \mathrm{MHz}, \mathrm{CDCl}_{3}\right): \delta(\mathrm{ppm}): 1.08\left(\mathrm{~s}, 6 \mathrm{H}, 2 \mathrm{CH}_{3}\right), 1.19\left(\mathrm{~s}, 6 \mathrm{H}, 2 \mathrm{CH}_{3}\right)$, 2.36-2.41 (m, 8H, 4CH $), 5.47(\mathrm{~s}, 1 \mathrm{H}, \mathrm{CH}), 6.20(\mathrm{~s}, 1 \mathrm{H}, \mathrm{OH}), 7.01-7.11\left(\mathrm{~m}, 4 \mathrm{H}, \mathrm{CH}_{\text {arom }}\right), 11.85$ $(\mathrm{s}, 1 \mathrm{H}, \mathrm{OH}) ;{ }^{13} \mathrm{C} \mathrm{NMR}\left(75 \mathrm{MHz}, \mathrm{CDCl}_{3}\right): \delta(\mathrm{ppm}) 26\left(2 \mathrm{CH}_{3}\right), 31\left(2 \mathrm{CH}_{3}\right), 32(2 \mathrm{Cq}), 33(\mathrm{CH})$, 47( $\left.2 \mathrm{CH}_{2}\right), 50\left(2 \mathrm{CH}_{2}\right), 117(2 \mathrm{Cq}), 119-129-136-157\left(6 \mathrm{C}_{\text {arom }}\right), 163(2=\mathrm{C}-\mathrm{OH}), 191(2 \mathrm{C}=\mathrm{O})$. E.I. $(70 \mathrm{eV}), \mathrm{m} / \mathrm{z}(\%): \mathrm{M}^{+.}=384(23), 246(57)$.

\section{3,3,6,6,9-pentamethyl-3,4,5,6,7,9-hexahydro-1H-xanthene-1,8(2H)-dione $\underline{4 a}$}

Yield: 67\%; ${ }^{1} \mathrm{H}$ NMR $\left(300 \mathrm{MHz}, \mathrm{CDCl}_{3}\right): \delta(\mathrm{ppm}): 1.04\left(\mathrm{~s}, 6 \mathrm{H}, 2 \mathrm{CH}_{3}\right), 1.09\left(\mathrm{~s}, 6 \mathrm{H}, 2 \mathrm{CH}_{3}\right)$, $1.24\left(\mathrm{~d}, \mathrm{~J}=6.1 \mathrm{~Hz}, 3 \mathrm{H}, \mathrm{CH}_{3}\right), 2.01-2.13\left(\mathrm{~m}, 8 \mathrm{H}, 4 \mathrm{CH}_{2}\right), 4.57(\mathrm{~d}, \mathrm{~J}=6.1 \mathrm{~Hz}, 1 \mathrm{H}, \mathrm{CH}) ;{ }^{13} \mathrm{C} \mathrm{NMR}$ ( $\left.75 \mathrm{MHz}, \mathrm{CDCl}_{3}\right): \delta(\mathrm{ppm}) 19\left(\mathrm{CH}_{3}\right), 22\left(2 \mathrm{CH}_{3}\right), 25\left(2 \mathrm{CH}_{3}\right), 28(2 \mathrm{Cq}), 30(\mathrm{CH}), 41\left(2 \mathrm{CH}_{2}\right)$, 43(2 $\left.\mathrm{CH}_{2}\right), 111(2 \mathrm{Cq}), 157(2=\mathrm{C}-\mathrm{O}), 183(2 \mathrm{C}=\mathrm{O})$. E.I. $(70 \mathrm{eV}), \mathrm{m} / \mathrm{z}(\%): \mathrm{M}^{7^{+}}=288(33), 166$ 
(37).

\section{3,3,6,6-tetramethyl-9-phenyl-3,4,5,6,7,9-hexahydro-1H-xanthene-1,8(2H)-dione $\underline{4 b}$}

Yield: 88\%; ${ }^{1} \mathrm{H}$ NMR $\left(300 \mathrm{MHz}, \mathrm{CDCl}_{3}\right): \delta(\mathrm{ppm}): 1.01\left(\mathrm{~s}, 6 \mathrm{H}, 2 \mathrm{CH}_{3}\right), 1.09\left(\mathrm{~s}, 6 \mathrm{H}, 2 \mathrm{CH}_{3}\right)$, 2.21-2.31 (m, 8H, 4CH $), 4.59(\mathrm{~s}, 1 \mathrm{H}, \mathrm{CH}), 7.01-7.24\left(\mathrm{~m}, 5 \mathrm{H}, \mathrm{CH}_{\text {arom }}\right) ;{ }^{13} \mathrm{C} \mathrm{NMR}(75 \mathrm{MHz}$, $\left.\mathrm{CDCl}_{3}\right): \delta(\mathrm{ppm}) 25\left(2 \mathrm{CH}_{3}\right), 30\left(2 \mathrm{CH}_{3}\right), 32(2 \mathrm{Cq}), 34(\mathrm{CH}), 45\left(2 \mathrm{CH}_{2}\right), 49\left(2 \mathrm{CH}_{2}\right)$, 123-125-127-154(6C $\mathrm{Carom}), 114(2 \mathrm{Cq}), 159(2=\mathrm{C}-\mathrm{O}), 184(2 \mathrm{C}=\mathrm{O})$. E.I. $(70 \mathrm{eV}), \mathrm{m} / \mathrm{z}(\%): \mathrm{M}\urcorner^{+}$. $=350(45), 228(7)$

\section{3,3,6,6-tetramethyl-9-(p-tolyl)-3,4,5,6,7,9-hexahydro-1H-xanthene-1,8(2H)-dione $\underline{4 c}$}

Yield: 74\%; ${ }^{1} \mathrm{H}$ NMR $\left(300 \mathrm{MHz}, \mathrm{CDCl}_{3}\right): \delta(\mathrm{ppm}): 1.03\left(\mathrm{~s}, 6 \mathrm{H}, 2 \mathrm{CH}_{3}\right), 1.11\left(\mathrm{~s}, 6 \mathrm{H}, 2 \mathrm{CH}_{3}\right)$, 2.15-2.27 (m, 8H, 4CH $), 2.47\left(\mathrm{~s}, 3 \mathrm{H}, \mathrm{CH}_{3}\right), 4.72(\mathrm{~s}, 1 \mathrm{H}, \mathrm{CH}), 6.86-7.24\left(\mathrm{~m}, 4 \mathrm{H}, \mathrm{CH}_{\text {arom }}\right)$; ${ }^{13} \mathrm{C}$ NMR ( $\left.75 \mathrm{MHz}, \mathrm{CDCl}_{3}\right): \delta(\mathrm{ppm}) 24\left(\mathrm{CH}_{3}\right.$ arom $), 26\left(2 \mathrm{CH}_{3}\right), 28\left(2 \mathrm{CH}_{3}\right), 30(2 \mathrm{Cq}), 31$ $(\mathrm{CH}), 41\left(2 \mathrm{CH}_{2}\right), 43\left(2 \mathrm{CH}_{2}\right), 110(2 \mathrm{Cq}), 125-126-131-152\left(6 \mathrm{C}_{\text {arom }}\right), 160(2=\mathrm{C}-\mathrm{O}), 189(2 \mathrm{C}=\mathrm{O})$. E.I. $(70 \mathrm{eV}), \mathrm{m} / \mathrm{z}(\%): \mathrm{M}\urcorner^{+.}=364(38), 242(51)$.

3,3,6,6-tetramethyl-9-(4-nitrophenyl)-3,4,5,6,7,9-hexahydro-1H-xanthene-1,8(2H)-dione $\underline{4 d}$

Yield: $96 \% ;{ }^{1} \mathrm{H}$ NMR $\left(300 \mathrm{MHz}, \mathrm{CDCl}_{3}\right): \delta(\mathrm{ppm}): 1.02\left(\mathrm{~s}, 6 \mathrm{H}, 2 \mathrm{CH}_{3}\right), 1.15\left(\mathrm{~s}, 6 \mathrm{H}, 2 \mathrm{CH}_{3}\right)$, 2.15-2.46 (m, 8H, 4CH $), 4.74(\mathrm{~s}, 1 \mathrm{H}, \mathrm{CH}), 7.39-8.06\left(\mathrm{~m}, 4 \mathrm{H}, \mathrm{CH}_{\text {arom }}\right) ;{ }^{13} \mathrm{C} \mathrm{NMR}(75 \mathrm{MHz}$, $\left.\mathrm{CDCl}_{3}\right): \delta(\mathrm{ppm}) 26\left(2 \mathrm{CH}_{3}\right), 30\left(2 \mathrm{CH}_{3}\right), 33(2 \mathrm{Cq}), 35(\mathrm{CH}), 47\left(2 \mathrm{CH}_{2}\right), 51\left(2 \mathrm{CH}_{2}\right), 114(2 \mathrm{Cq})$, 121-127-140-155(6C $\left.\mathrm{C}_{\text {arom }}\right), 162(2=\mathrm{C}-\mathrm{O}), 185(2 \mathrm{C}=\mathrm{O})$. E.I. $(70 \mathrm{eV}), \mathrm{m} / \mathrm{z}(\%): \mathrm{M}^{++}=395(39)$, 273(65).

9-(4-fluorophenyl)-3,3,6,6-tetramethyl-3,4,5,6,7,9-hexahydro-1H-xanthene-1,8(2H)-dione $\underline{4 e}$

Yield: 93\%; ${ }^{1} \mathrm{H}$ NMR $\left(300 \mathrm{MHz}, \mathrm{CDCl}_{3}\right): \delta(\mathrm{ppm}): 1.01\left(\mathrm{~s}, 6 \mathrm{H}, 2 \mathrm{CH}_{3}\right), 1.14\left(\mathrm{~s}, 6 \mathrm{H}, 2 \mathrm{CH}_{3}\right)$, 2.31-2.42 (m, 8H, 4CH $), 4.69(\mathrm{~s}, 1 \mathrm{H}, \mathrm{CH}), 6.91-7.04\left(\mathrm{~m}, 4 \mathrm{H}, \mathrm{CH}_{\mathrm{arom}}\right) ;{ }^{13} \mathrm{C} \mathrm{NMR}(75 \mathrm{MHz}$, $\left.\mathrm{CDCl}_{3}\right): \delta(\mathrm{ppm}) 25\left(2 \mathrm{CH}_{3}\right), 28\left(2 \mathrm{CH}_{3}\right), 31(2 \mathrm{Cq}), 33(\mathrm{CH}), 45\left(2 \mathrm{CH}_{2}\right), 49\left(2 \mathrm{CH}_{2}\right), 113(2 \mathrm{Cq})$, 117-125-131-157(6C $\left.\mathrm{C}_{\text {arom }}\right), 160(2=\mathrm{C}-\mathrm{O}), 187(2 \mathrm{C}=\mathrm{O})$. E.I. $(70 \mathrm{eV}), \mathrm{m} / \mathrm{z}(\%): \mathrm{M}^{++}=368(32)$, $246(43)$.

Methyl-4-(3,3,6,6-tetramethyl-1,8-dioxo-2,3,4,5,6,7,8,9-octahydro-1H-xanthen-9-yl)benzo ate $\underline{4 \mathbf{f}}$ 
Yield: 97\%; ${ }^{1} \mathrm{H}$ NMR $\left(300 \mathrm{MHz}, \mathrm{CDCl}_{3}\right): \delta(\mathrm{ppm}): 1.02\left(\mathrm{~s}, 6 \mathrm{H}, 2 \mathrm{CH}_{3}\right), 1.13\left(\mathrm{~s}, 6 \mathrm{H}, 2 \mathrm{CH}_{3}\right)$, 2.33-2.46 (m, 8H, 4CH $), 3.86\left(\mathrm{~s}, 3 \mathrm{H}, \mathrm{O}-\mathrm{CH}_{3}\right), 4.71(\mathrm{~s}, 1 \mathrm{H}, \mathrm{CH}), 7.14-7.93\left(\mathrm{~m}, 4 \mathrm{H}, \mathrm{CH}_{\text {arom }}\right)$; ${ }^{13} \mathrm{C} \mathrm{NMR}\left(75 \mathrm{MHz}, \mathrm{CDCl}_{3}\right): \delta(\mathrm{ppm}) 27\left(2 \mathrm{CH}_{3}\right), 32\left(2 \mathrm{CH}_{3}\right), 34(2 \mathrm{Cq}), 35(\mathrm{CH}), 48\left(2 \mathrm{CH}_{2}\right)$, $49\left(\mathrm{O}-\mathrm{CH}_{3}\right), \quad 53\left(2 \mathrm{CH}_{2}\right), \quad 118(2 \mathrm{Cq}), \quad 129-130-131-159\left(6 \mathrm{C}_{\text {arom }}\right), \quad 163(2=\mathrm{C}-\mathrm{O}), \quad 167(\mathrm{Cq})$, 192(2C=O). E.I. $(70 \mathrm{eV}), \mathrm{m} / \mathrm{z}(\%): \mathrm{M}^{+.}=348(15)$.

9-(4-hydroxyphenyl)-3,3,6,6-tetramethyl-3,4,5,6,7,9-hexahydro-1H-xanthene-1,8(2H)-dio ne $4 \mathrm{~g}$

Yield: $81 \%$; ${ }^{1} \mathrm{H}$ NMR $\left(300 \mathrm{MHz}, \mathrm{CDCl}_{3}\right): \delta(\mathrm{ppm}): 1.01\left(\mathrm{~s}, 6 \mathrm{H}, 2 \mathrm{CH}_{3}\right), 1.11\left(\mathrm{~s}, 6 \mathrm{H}, 2 \mathrm{CH}_{3}\right)$, 2.31-2.39 (m, 8H, 4CH $), 4.57(\mathrm{~s}, 1 \mathrm{H}, \mathrm{CH}), 6.20(\mathrm{~s}, 1 \mathrm{H}, \mathrm{OH}), 6.69-7.08\left(\mathrm{~m}, 4 \mathrm{H}, \mathrm{CH}_{\text {arom }}\right)$; ${ }^{13} \mathrm{C}$ NMR ( $\left.75 \mathrm{MHz}, \mathrm{CDCl}_{3}\right): \delta(\mathrm{ppm}) 26\left(2 \mathrm{CH}_{3}\right), 31\left(2 \mathrm{CH}_{3}\right), 32(2 \mathrm{Cq}), 33(\mathrm{CH}), 47\left(2 \mathrm{CH}_{2}\right)$, 50(2 $\left.\mathrm{CH}_{2}\right), 117(2 \mathrm{Cq}), 119-129-136-157\left(6 \mathrm{C}_{\mathrm{arom}}\right), 163(2=\mathrm{C}-\mathrm{O}), 191(2 \mathrm{C}=\mathrm{O})$. E.I. $(70 \mathrm{eV}), \mathrm{m} / \mathrm{z}$ $(\%): \mathrm{M}^{++}=366(32), 244(43)$.

9-(4-fluorophenyl)-3,3,6,6,10-pentamethyl-3,4,6,7,9,10-hexahydroacridine-1,8(2H,5H)-di one $\underline{5 a}$

Yield: 45\%; ${ }^{1} \mathrm{H}$ NMR $\left(300 \mathrm{MHz}, \mathrm{CDCl}_{3}\right): \delta(\mathrm{ppm}): 0.98\left(\mathrm{~s}, 6 \mathrm{H}, 2 \mathrm{CH}_{3}\right), 1.07\left(\mathrm{~s}, 6 \mathrm{H}, 2 \mathrm{CH}_{3}\right)$, 2.15-2.33 (m, 8H, 4CH 2 ), 2.95(s, 3H, N-CH 3 ), 5.07 (s, 1H, CH), 6.91-7.02 (m, 4H, $\mathrm{CH}_{\text {arom }}$ ); ${ }^{13} \mathrm{C} \mathrm{NMR}\left(75 \mathrm{MHz}, \mathrm{CDCl}_{3}\right): \delta(\mathrm{ppm}) 26\left(2 \mathrm{CH}_{3}\right), 32\left(2 \mathrm{CH}_{3}\right), 29(2 \mathrm{Cq}), 34(\mathrm{CH}), 44\left(2 \mathrm{CH}_{2}\right)$, $47\left(2 \mathrm{CH}_{2}\right), 112(2 \mathrm{Cq}), 116-129-134-158\left(6 \mathrm{C}_{\text {arom }}\right), 161(2=\mathrm{C}-\mathrm{N}), 191(2 \mathrm{C}=\mathrm{O})$. C.I. $\left.\left(\mathrm{NH}_{3}\right): \mathrm{MH}\right\urcorner^{+}$ $=382(41 \%), 244(43 \%)$.

9-(4-fluorophenyl)-3,3,6,6-tetramethyl-10-phenyl-3,4,6,7,9,10-hexahydroacridine-1,8(2H, 5H)-dione $\underline{5 b}$

Yield: 63\%; ${ }^{1} \mathrm{H}$ NMR $\left(300 \mathrm{MHz}, \mathrm{CDCl}_{3}\right): \delta(\mathrm{ppm}): 0.97\left(\mathrm{~s}, 6 \mathrm{H}, 2 \mathrm{CH}_{3}\right), 1.08\left(\mathrm{~s}, 6 \mathrm{H}, 2 \mathrm{CH}_{3}\right)$, 2.17-2.36 (m, 8H, 4CH $), 5.06(\mathrm{~s}, 1 \mathrm{H}, \mathrm{CH}), 6.77-7.41\left(\mathrm{~m}, 9 \mathrm{H}, \mathrm{CH}_{\text {arom }}\right) ;{ }^{13} \mathrm{C} \mathrm{NMR}(75 \mathrm{MHz}$, $\left.\mathrm{CDCl}_{3}\right): \delta(\mathrm{ppm}) 27\left(2 \mathrm{CH}_{3}\right), 32\left(2 \mathrm{CH}_{3}\right), 31(2 \mathrm{Cq}), 33(\mathrm{CH}), 46\left(2 \mathrm{CH}_{2}\right), 49\left(2 \mathrm{CH}_{2}\right), 113(2 \mathrm{Cq})$, 115-117-118-124-128-133-134-156(12C arom $), 163(2=\mathrm{C}-\mathrm{N}), 192(2 \mathrm{C}=\mathrm{O})$. C.I. $\left(\mathrm{NH}_{3}\right): \mathrm{MH}^{+}=$ $444(37 \%), 231(29 \%)$.

Methyl-4-(3,3,6,6,10-pentamethyl-1,8-dioxo-1,2,3,4,5,6,7,8,9,10-decahydroacridin-9-yl)be nzoate $\underline{\mathbf{5 c}}$

Yield: $51 \%$; ${ }^{1} \mathrm{H}$ NMR $\left(300 \mathrm{MHz}, \mathrm{CDCl}_{3}\right): \delta(\mathrm{ppm}): 0.99\left(\mathrm{~s}, 6 \mathrm{H}, 2 \mathrm{CH}_{3}\right), 1.12\left(\mathrm{~s}, 6 \mathrm{H}, 2 \mathrm{CH}_{3}\right)$, 
2.31-2.39 (m, 8H, 4CH$), 3.01\left(\mathrm{~s}, 3 \mathrm{H}, \mathrm{N}-\mathrm{CH}_{3}\right), 3.83\left(\mathrm{~s}, 3 \mathrm{H}, \mathrm{O}-\mathrm{CH}_{3}\right), 5.12(\mathrm{~s}, 1 \mathrm{H}, \mathrm{CH})$, 7.09-7.88 (m, 4H, $\left.\mathrm{CH}_{\text {arom }}\right) ;{ }^{13} \mathrm{C} \mathrm{NMR}\left(75 \mathrm{MHz}, \mathrm{CDCl}_{3}\right): \delta(\mathrm{ppm}) 25\left(2 \mathrm{CH}_{3}\right), 33\left(2 \mathrm{CH}_{3}\right), 34$ $(2 \mathrm{Cq}), \quad 36(\mathrm{CH}), \quad 39\left(\mathrm{~N}-\mathrm{CH}_{3}\right), \quad 46\left(\mathrm{O}-\mathrm{CH}_{3}\right), \quad 48\left(2 \mathrm{CH}_{2}\right), \quad 55\left(2 \mathrm{CH}_{2}\right), \quad 114(2 \mathrm{Cq})$, 126-129-131-159(6C $\left.\mathrm{C}_{\text {arom }}\right), 165(2=\mathrm{C}-\mathrm{N}), 167(\mathrm{Cq}), 193(2 \mathrm{C}=\mathrm{O})$. E.I. $\left.(70 \mathrm{eV}), \mathrm{m} / \mathrm{z}(\%): \mathrm{M}\right\urcorner^{+.}=$ 390 (43), 244(39).

Methyl-4-(3,3,6,6-tetramethyl-1,8-dioxo-10-phenyl-1,2,3,4,5,6,7,8,9,10-decahydroacridin-

\section{9-yl)benzoate $\underline{5 \mathrm{~d}}$}

Yield: 67\%; ${ }^{1} \mathrm{H}$ NMR $\left(300 \mathrm{MHz}, \mathrm{CDCl}_{3}\right): \delta(\mathrm{ppm}): 1.09\left(\mathrm{~s}, 6 \mathrm{H}, 2 \mathrm{CH}_{3}\right), 1.21\left(\mathrm{~s}, 6 \mathrm{H}, 2 \mathrm{CH}_{3}\right)$, 2.33-2.41 (m, 8H, 4CH $), 3.87\left(\mathrm{~s}, 3 \mathrm{H}, \mathrm{O}-\mathrm{CH}_{3}\right), 5.11(\mathrm{~s}, 1 \mathrm{H}, \mathrm{CH}), 6.89-7.91\left(\mathrm{~m}, 9 \mathrm{H}, \mathrm{CH}_{\text {arom }}\right)$; ${ }^{13} \mathrm{C} \mathrm{NMR}\left(75 \mathrm{MHz}, \mathrm{CDCl}_{3}\right): \delta(\mathrm{ppm}) 26\left(2 \mathrm{CH}_{3}\right), 31\left(2 \mathrm{CH}_{3}\right), 35(2 \mathrm{Cq}), 37(\mathrm{CH}), 47\left(\mathrm{O}_{-} \mathrm{CH}_{3}\right)$, $49\left(2 \mathrm{CH}_{2}\right), 56\left(2 \mathrm{CH}_{2}\right), 114(2 \mathrm{Cq}), 116-119-123-128-130-132-134-159\left(12 \mathrm{C}_{\text {arom }}\right), 162(2=\mathrm{C}-\mathrm{N})$, 168(Cq), 194(2C=O).E.I. $(70 \mathrm{eV}), \mathrm{m} / \mathrm{z}(\%): \mathrm{M}\urcorner^{+.}=466(49), 308(76)$.

\section{CONCLUSION}

In this work, we achieved the synthesis of a new type of dihydropyridinemolecules $\underline{\mathbf{5}}$ under clean operating conditions: $\mathrm{H}_{2} \mathrm{O}$ as a solvent, no-catalystsand a simply work-up by filtration. The selectivity formation of structures $\underline{\mathbf{3}}$ and $\underline{\mathbf{4}}$, obtained with good yields, is determined to be conditioned by the reaction time.

\section{ACKNOWLEDGEMENTS}

The authors would like to think the Department of Organic Chemistry. Laboratoire de Chimie Organique et des Substances Naturelles, Université Ziane Achour, Djelfa, Algerie and the Faculté de Chimie - USTHB - BP32, El-Alia, 16111 Bab Ezzouar, Alger, Algerie ; Universidad de Valencia and the Instituto de Reconocimiento Molecular y Desarrollo Tecnológico and Centro Mixto Universidad Politécnica de Valencia-Universidad de Valencia. Spain.

\section{REFERENCES}

[1] Appendino, G.; Cicioni, L.; Minassi, A. [A multicomponent synthesis of 
gem-( $\beta$-dicarbonyl)arylmethanes], Tetrahedron Lett., 2009, 50, 5559-5561.

[2] Rosa, A.; Deiana, M.; Atzeri, A.; Corona, G.; Incani, A.; Melis, M.P.; Appendino, G.; Dessi, M.A. [Evaluation of the antioxidant and cytotoxic activity of arzanol, a prenylated alpha-pyrone-phloroglucinol etherodimer from Helichrysum italicum subsp.microphyllum], Chem. Biol. Interact., 2007, 165, 117- 126.

[3] Sinkkonen, J.; Ovcharenko, V.; Zelenin, K. N.; Bezhan, I. P.; Chakchir, B. A.; Al-Assar, F.; Pihlaja, K. [ ${ }^{1} \mathrm{H}$ and ${ }^{13} \mathrm{C}$ NMR Study of 1-Hydrazino-2,3-dihydro-1H-pyrazolo[1,2- $\alpha$ ] pyridazine-5,8-diones and -1H-pyrazolo[1,2-b]phthalazine-5,10-diones and Their Ring-Chain Tautomerism], Eur. J. Org. Chem., 2002, 13, 2046-2053.

[4] Kozlov, N.G.; Kadutskii, A. P. [A novel three-component reaction of anilines, formaldehyde and dimedone: simple synthesis of spirosubstituted piperidines], Tetrahedron Lett., 2008, 49, 4560-4562

[5] Donelson, J. L.; Gibbs, R. A.; De, S. K. [An efficient one-pot synthesis of polyhydroquinoline derivatives through the Hantzsch four component condensation], J. Mol. Catal. A :Chem. 256, 2006, 309-311.

[6] Xiao, W.; Wei-Wei M.; Li-Qiang, W.; Fu-Lin, Y. [Synthesis of 2H-Indazolo[2,1-b]phthalazine-1,6,11(13H)-trione Derivatives Using Wet Cyanuric Chloride under Solvent-Free Condition], J. Chinese Chemical Society, 2010, 57, 1341-1345

[7] Shi, D. Q.; Wang, Y.H.; Lu, Z.S.; Dai, G.Y. [Condensation of aromatic aldehydes with acidic methylene compounds without catalyst], Synth. Commun., 2000, 30, 713-726

[8] Suarez, M.; Ochoa, E.; Verdecia, Y.; Pita, B.; Moran, L.; Martin, N.; Quinteiro, M.; Seoane, C.; Soto, J.L.; Novoa, H.; Blaton, N.; Peters, O.M. [A joint experimental and theoretical structural study of novel substituted2,5-dioxo-1,2,3,4,5,6,7,8-octahydroquinolines], Tetrahedron, 1999, 55, 875-884.

[9] Hassani, Z.; Islami, M.R.; Kalantari, M. [An efficient one-pot synthesis of octahydroquinazolinone derivatives using catalytic amount of $\mathrm{H}_{2} \mathrm{SO}_{4}$ in water], Bioorg. Med. Chem. Lett., 2006,16, 4479-4482.

[10] Majumdar, K.C.; Samanta, S.K. [Studies in aza-Claisen rearrangement: synthesis of dimedone-annelated unusual heterocycles], Tetrahedron, 2001, 57, 4955 - 4958. 
[11] Quirago, J.; Mejia, D.; Insuasty, B.; Abonia, R.; Nogueras, M.; Sanchez, A.; Cobo, J.; Low, J.N. [Regioselective synthesis of 4,7,8,9-tetrahydro-2H-pyrazolo[3,4-b]quinolin-5 (6H)ones. Mechanism and structural analysis], Tetrahedon, 2001, 57, 6947 - 6953.

[12] Devi, I.; Bhuyan, P.J. [Sodium bromide catalysed one-pot synthesis of tetrahydrobenzo[b]pyrans via a three-component cyclocondensation under microwave irradiation and solvent free conditions], Tetrahedron Lett., 2004, 45, 8625-8627.

[13] Lai, J.T.; Kuo, P.Y.; Gau, Y.H.; Yang, D.Y. [Synthesis and characterization of coumarin and dimedone-derived diazabicycles], Tetrahedron Lett., 2007, 48, 7796-7800.

[14] Kantevari, S.; Bantu, R.; Nagarapu, L. $\left[\mathrm{HClO}_{4}-\mathrm{SiO}_{2}\right.$ and PPA-SiO 2 catalyzed efficient one-pot Knoevenagel condensation, Michael addition and cyclo-dehydration of dimedone and aldehydes in acetonitrile, aqueous and solvent free conditions: scope and limitations], J. Mol. Catal.A: Chem. 2007, 269, $53-57$

[15] Bouaziz, O.; Abboub, F.; Khier-Bayou, N.; Fodili, M.; Hoffmann, P.; Amari, M. [Réactivité de l'acide déhydroacétique hydrogéné en $\mathrm{C}_{5}-\mathrm{C}_{6}$ : Obtention des pyrano-1,5-benzodiazépines différemment substituées et de la structure énaminone]. $C$. $R$. Chimie, 2012, 15, 774-778.

[16] Fodili, M.; Hoffmann, P.; Amari, M. [Synthesis of 4-pyrano-1,5-benzodiazepines catalyzed by bismuth (III) derivatives], Research Journal of Pharmaceutical, Biological and Chemical Sciences, RJPBCS, 2012, 3, 10-15.

[17] Fodili, M.; Bellara, N. K.; Garrigues, B.; Lebert, C.; Hoffmann, P. [Synthesis of Imidazoles from Ketimines Using Tosylmethyl Isocyanide (TosMIC) Catalyzed by Bismuth Triflate], Lett. Org. Chem., 2009, 6, 354-358.

[18] Kadutskii, A. P.; Kozlov, N. G. [A novel three-component reaction of anilines, formaldehyde and $\beta$-Diketones: Simple synthesis of 3-spirosubstituted 1,2,3,4-tetrahydroquinolines], Synlett., 2006, 19, 3349-3351.

\section{How to cite this article:}

Bayou-Khier N, Amari M, Fodili M, Grau SG, Hoffmann P. A green and efficient method for the synthesis of homodimeric ( $\beta$-dicarbonyl) arylmethanes and dihydropyridine from dimedone in water. J. Fundam. Appl. Sci., 2016, 8(3), 945-955. 